\title{
Unobtrusive Monitoring of ECG and EEG During Mild Stress Stimuli
}

\author{
Veronica C. Zuccalà ${ }^{1,2}$, Riccardo Favilla ${ }^{2}$, Giuseppe Coppini ${ }^{2}$ \\ ${ }^{1}$ Alma Mater Studiorum, University of Bologna, Bologna, Italy \\ ${ }^{2}$ Institute of Clinical Physiology of the National Research Council, Pisa, Italy
}

\begin{abstract}
This study is part of a research project aiming to build a model for quantifying an individual wellness status through unobtrusive measurements of psychophysical parameters and self-reported data. In particular, we focus on the evaluation of the individual response to mild stress stimuli. The experimental setup included: EG05000 Medlab Five Channel Module, Gigabit Ethernet camera, BioHarness 3 Zephyr chest belt, and Muse 2 Headband. Experimental results show increased heart rate and respiration rate, and changes of the brain activity in the stress condition. This is consistent with a "fight or flight" response in accordance with literature. Therefore, the methodology applied in this study can be used to monitor the individual wellness status in conditions of mild stress stimuli.
\end{abstract}

\section{Introduction}

Stress is usually a state of tension that is created when a person responds to demands and pressures arising from external sources (e.g. work, family, and social environment) as well as those internally generated (e.g. self-imposed demands and obligations, self-criticism) [1]. Stress is a common state of emotional strain that plays a crucial role in everyday quality of life. This state consists of several complementary and interacting components (i.e. cognitive, affective, and psycho-physiological). Furthermore, chronic stress carries a wild range of healthrelated diseases, including cardiovascular diseases, cerebrovascular diseases, diabetes, and immune deficiencies [2].

Due to the adverse effects of stress in our daily life, stress management has been receiving an increasing attention in health-care and well-being research [3]. Moreover, there is a growing individual awareness of the importance of a proper lifestyle and a generalized trend to become an active part in monitoring, preserving, and improving personal wellness. In this respect, stress related factors, together with the development of personalized coping strategies, are of paramount importance.

In recent years, a growing number of studies addressed the development of systems for quantifying the individual health and wellness status [4]. In particular, being stress a complex multifactorial process, one needs to identify a contained set of parameters suitable for its detection and description.

As described in [3], the autonomic nervous systems (ANS) controls the organs of our body such as the heart, the stomach, and the intestines. ANS includes sympathetic and parasympathetic nervous systems. The parasympathetic nervous system is responsible for nourishing, calming the nerves to return to the regular function, healing, and regeneration. On the contrary, the sympathetic system is accountable for activating the glands and the organs for defending the body from the threat. The activation of the sympathetic nervous system might be accompanied by many physical reactions, such as an increase in the heart rate (HR), rapid blood flow to the muscle, activation of sweat glands, and increase in the respiratory rate. In addition, the electroencephalograms (EEG), which reflect brain activity, are also important for detecting and assessing mental stress [5]. Some neurophysiological studies have reported the relationship between human emotion and hemispheric specialization, where the left hemisphere is more involved in processing positive emotions, and the right hemisphere is more involved in processing negative emotions. Furthermore, the prefrontal cortex accounts for a large proportion of emotional processing. Stress usually causes negative moods, such as depression, anger, and anxiety, resulting in increased right-prefrontal activity. Thus, the asymmetric analysis of the frequency-band powers in the EEG measured at the prefrontal cortex has been generally applied in previous stress studies.

The majority of clinical studies use the EEG channels from hair-bearing scalp areas of the 10-20 systems. However, this method requires the use of a conductive gel and an appropriate preparation procedure, which are particularly inconvenient for users. Indeed, the EEG recordings from hairless regions such as the forehead, or behind or inside the ear, would be more suitable for longterm monitoring in daily life.

In terms of achieving continuous stress management in daily life, device usability is the key so we defined an experimental setup based on the analysis of physiological parameters during mild stress stimuli.

In accordance with these considerations, we have defined an experimental setup based on the integration of simple unobtrusive devices monitoring the heart rate, the 
respiratory rate and the EEG signal. The devices were used to monitor the individual response in a resting state and during mild stress stimuli.

\section{Methods}

\subsection{Study's participants}

Seven healthy participants ( 5 females and 2 males with mean age of 41.72 years, range from 25 to 62) were recruited for voluntary participation in this study. The experimental protocol received the Ethical Clearance certification (0050349/2019, July 09th, 2019) by the National Research Council Committee for Research Ethics and Bioethics. Written informed consent was obtained from all subjects included in this study.

\subsection{Experimental setup}

The following set of commercial devices were used:

- EG05000 Medlab ECG Five Channel Module (Medlab GmbH, DE) operating at $300 \mathrm{~Hz}$ was employed to acquire the ECG lead sampled at $300 \mathrm{~Hz}$.

- Gigabit Ethernet camera with a CMOS monochrome sensor (UI-55240SE-NIR-GL, IDS $\mathrm{GmbH}, \mathrm{DE})$. The camera was operated at $133 \mathrm{fps}$ with an image matrix of 352 x 224 pixel, 8 bits/pixel. The video images were saved in real time in raw format by a Mac mini system (Intel Core i7 dual-core processor with $3 \mathrm{GHz}, 16 \mathrm{~GB}$ RAM, and 500 GB SSD, Apple Inc USA). In order to enhance the plethysmographic signal, the camera mounted an optical band-pass filter centered at $550 \mathrm{~nm}$ with a bandwidth of $40 \mathrm{~nm}$.

- BioHarness 3 Zephyr chest belt (Medtronic Inc, USA) for measuring the respiratory rate.

- Muse 2 Headband (InteraXon Inc, USA) for the EEG signal acquisition.

The subjects were sitting still in front of the computer monitor at a distance of about one meter. The chair had a headrest to contain head motion and make the recording comfortable for the volunteer. The subjects were illuminated by a white LED light source. At first the volunteer was asked to provide base data including: age, sex, weight, height, current perceived stress level through the Perceived Stress Scale (PSS) [6], and physical activity through Rapid Assessment of Physical Activity questionnaire (RAPA) [7]. Subsequently, all the devices were positioned and calibrated. At the beginning (T0), the subject's signals were recorded for 5 minutes in resting state, subsequently (T1), signals were acquired during a mild stress stimulation. At T0, the subject was asked to close his/her eyes and relax. At T1 stress was induced by the Stroop Color Word Test (SCWT) [8]. A portable version of the Stroop test was implemented by an ad hoc Java app. The subject played a congruent version of the SCWT, where the font color always matched the displayed color name. The time limit to answer each question was set to two seconds and the overall test lasted two minutes. At the end of the test, a short questionnaire was filled out by the volunteer to assess the stress level experienced by the subject. Stress self-assessment was conducted using two different questions as reported by Giokoumis et al. [9]. The first one was a Likert-scaled (15) question directly asking subjects whether they were feeling stressed during the test [10]. The second was a subset of the stress appraisal measure questionnaire [11] including questions $2,16,24$, and 26.

\subsection{ECG signal analysis}

The ECG signal was analyzed as described in [12]. The tachogram was firstly extracted from the ECG signals. To this end, Inter Beat Intervals (IBIs) were defined following QRS complex detection. To remove possible artifacts, the IBIs were processed by the NC-VT algorithm with a tolerance of $30 \%$, all the IBIs with a duration less than $200 \mathrm{~ms}$ being removed. The signals were analyzed both in time domain and in frequency domain. Concerning time domain, we calculated the average time between adjacent normal heartbeats (NN) and its standard deviation (SDNN). Concerning frequency domain, analysis of the heart rate variability (HRV) was performed by power spectrum density (PSD) estimated by the Lomb-Scargle periodogram. This method is able to cope with non-uniformly sampled data even in presence of large gaps, which makes the tachogram interpolation unnecessary. According to the standard definition of the HRV frequency bands, low frequency (LF) and high frequency $(\mathrm{HF})$ were calculated as the area under the PSD curve corresponding from $0.04 \mathrm{~Hz}$ to $0.15 \mathrm{~Hz}$ and from $0.15 \mathrm{~Hz}$ to $0.4 \mathrm{~Hz}$, respectively. The $\mathrm{LF}$ component reflects both sympathetic and parasympathetic actions, the $\mathrm{HF}$ component reflects parasympathetic action, and the $\mathrm{LF} / \mathrm{HF}$ ratio is a measure of the sympatho/vagal balance.

\subsection{Video signal analysis}

The video signal was analyzed as described in [12]. According to [12], for each video sequence, the Cascading Classifiers algorithm (OpenCV v. 3.0.0) was used to detect the face of the subject and locating the center of each eye in the first frame of the recorded video. To extract the heart rate and the heart rate variability parameters, image grey levels were averaged in the three automatically selected regions of interest (ROIs), which provided three time signals $\mathrm{x}_{1}(\mathrm{t}), \mathrm{x}_{2}(\mathrm{t})$, and $\mathrm{x}_{3}(\mathrm{t})$. They 
were de-trended by subtracting a $2 \mathrm{~s}$ long time average and were jointly processed through independent component analysis (ICA) as implemented in FastICA algorithm; three new sequences $\mathrm{y}_{1}(\mathrm{t}), \mathrm{y}_{2}(\mathrm{t})$, and $\mathrm{y}_{3}(\mathrm{t})$ were so obtained, each representing a different signal contribution. The spectra of $\mathrm{y}_{1}(\mathrm{t}), \mathrm{y}_{2}(\mathrm{t})$, and $\mathrm{y}_{3}(\mathrm{t})$ were analyzed. The component $\mathrm{ys}_{\mathrm{s}}(\mathrm{t})$ having the highest peak in the range from $0.75 \mathrm{~Hz}$ to $2.0 \mathrm{~Hz}$ was selected as blood volume pulse (BVP) component. The $y_{s}(t)$ was then filtered using a FIR band-pass filter implemented via Hamming window, with lower cut-off at $0.75 \mathrm{~Hz}$ $(45 \mathrm{bpm})$ and upper cut-off at $2 \mathrm{~Hz}(120 \mathrm{bpm})$. After filtering, BVP peaks were detected. Finally, the tachogram for each BVP peak sequence was computed. The NN and SDNN values were extracted from the tachogram. Analysis of the HRV was performed in the same way of ECG signal analysis.

\subsection{Respiratory rate signal analysis}

The respiratory rate was measured through BioHarness 3 Zephyr. The device provides the respiratory rate at $1 \mathrm{~Hz}$ sampling frequency. The values of the median and the interquartile range were calculated.
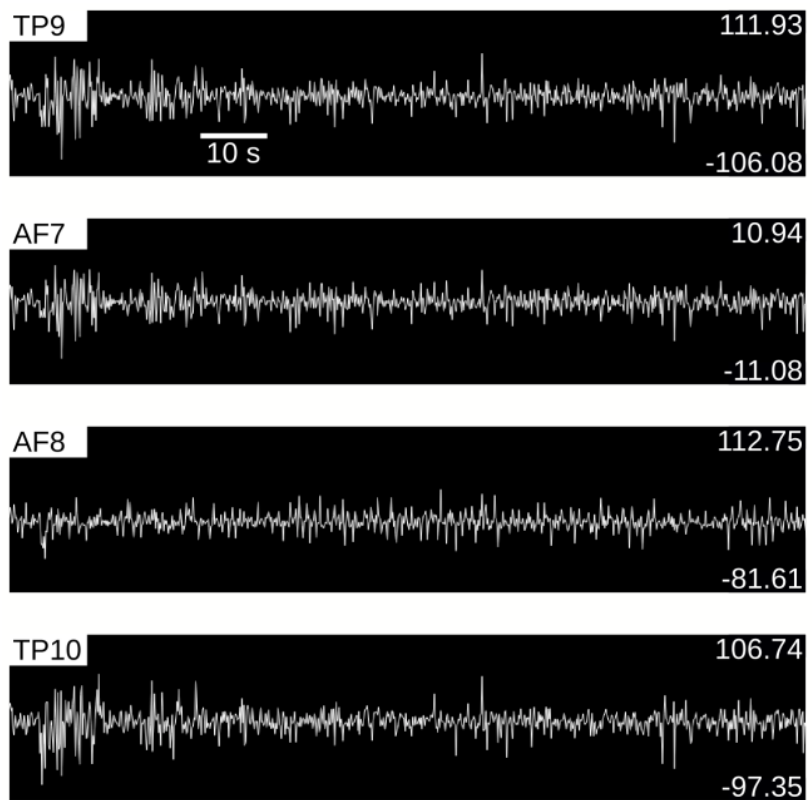

Figure 1. The EEG signals after ICA processing.

\subsection{EEG signal analysis}

The Muse 2 Headband is a simple electroencephalography device. It has three reference sensors on the forehead, two channels on the left (AF7 and TP9) and two on the right (AF8 and TP10). AF7 and AF8 are forehead sensors and TP9 and TP10 are
SmartSense conductive rubber ear sensors. The features extracted from the EEG [5] through a custom software written in $\mathrm{C}$ include the normalized band power (delta from $0.5 \mathrm{~Hz}$ to $4 \mathrm{~Hz}$, theta from $4 \mathrm{~Hz}$ to $8 \mathrm{~Hz}$, alpha from $8 \mathrm{~Hz}$ to $13 \mathrm{~Hz}$, beta from $13 \mathrm{~Hz}$ to $30 \mathrm{~Hz}$ ) for each channel, that is calculated as:

$$
\frac{\text { Summation of power from } f_{1} \text { to } f_{2} \mathrm{~Hz}}{\text { Total power }} \times 100
$$

and each band's (delta, theta, alpha, beta) power asymmetry (DPA, TPA, APA, BPA), that is calculated as:

$$
\frac{\text { EEG band power }_{R}-\text { EEG band power }}{L}
$$

The four signals were jointly processed through Independent Component Analysis as implemented in FastICA algorithm; four new sequences were so obtained, each representing a different signal contribution. One of these signals was identified as the one with eyes blinking and was removed. The remaining signals were analyzed through the inverse FastICA in order to obtain four signals again. The analysis was performed by power spectrum density estimated by the Lomb-Scargle periodogram.

\section{Results}

In Table I we report the median and the interquartile range of each parameter as observed in the study sample. Concerning the ECG results, the median value of the NN is $926.98 \mathrm{~ms}$ in $\mathrm{T} 0$ and $820.66 \mathrm{~ms}$ in T1. Concerning the video results, the median value of the $\mathrm{NN}$ is $851.74 \mathrm{~ms}$ in T0 and $822.67 \mathrm{~ms}$ in T1. The HRV parameters of the ECG have smaller variability in $\mathrm{T} 1$ than in T0. The Pearson correlation coefficient between the $\mathrm{NN}$ value of the ECG and the video signal is equal to 0.93 and 0.95 , respectively for $\mathrm{T} 0$ and $\mathrm{T} 1$ ( $\mathrm{p}$-value $<0.003$ ). The median value of the RR is $64 \mathrm{bpm}$ in T0 and $66 \mathrm{bpm}$ in T1. The most relevant feature extracted from the EEG is the power asymmetry from the left and right normalized alpha band power of AF7 and AF8 sensors. The APA was significantly smaller in $\mathrm{T} 1$ (median value $=-0.35$ ) than in T0 (median value $=0.27$ ) with $\mathrm{p}$-value $=0.01$. 
Table 1. For each condition, the median and the interquartile range, in brackets, of the all parameters extracted are reported.

\begin{tabular}{|c|c|c|}
\hline Parameter & $T 0$ & $T 1$ \\
\hline$N N_{E C G}(m s)$ & $926.98(128.78)$ & $820.66(191.41)$ \\
\hline$S D N N_{E C G}(m s)$ & $47.94(42.80)$ & $42.40(29.77)$ \\
\hline $\begin{array}{c}L F_{E C G} \\
(\text { normalized unit })\end{array}$ & $79.09(24.80)$ & $54.86(19.16)$ \\
\hline $\begin{array}{c}H F_{E C G} \\
(\text { normalized unit })\end{array}$ & $20.91(24.80)$ & $45.14(19.16)$ \\
\hline $\begin{array}{c}L F_{E C G} / H F_{E C G} \\
\text { NN }\end{array}$ VIDEO $(m s)$ & $851.74(144.62)$ & $822.67(272.95)$ \\
\hline$R R(b p m)$ & $64(9)$ & $66(11)$ \\
\hline$A P A$ & $0.27(0.65)$ & $-0.35(0.28)$ \\
\hline
\end{tabular}

\section{Discussion and Conclusion}

In this study we used three unobtrusive devices that are the Gigabit Ethernet camera, the BioHarness 3 Zephyr chest belt, and the Muse 2 Headband. Furthermore, the subjects involved in the study were submitted to mild stress stimuli. During the stress condition, the heart rate and the respiratory rate increased, and changes of the brain activity was observed. In particular the last factor indicates that the right alpha power was reduced to a greater extent than the left alpha power in a stress situation, which is consistent with the physiological assumptions (i.e., enhanced activation occurred in the right hemisphere, which shows negative emotions). The results obtained demonstrate that the devices and the method involved in this study allow detecting differences in a mild stress stimuli condition through unobtrusive measurements of psychophysical parameters and selfreported data. We are currently planning the acquisition of data from an extended sample of volunteers. Analysis of intra-subject differences will be also carried out on such expanded dataset. Furthermore, all the information that will be extracted will use to build a model for quantifying an individual wellness status.

\section{References}

[1] W. Liao, W. Zhang, Z. Zhu, and Q. Ji, "A real-time human stress monitoring system using dynamic Bayesian network," 2005 IEEE Computer Society Conference on Computer Vision and Pattern Recognition (CVPR'05)-Workshops, pp. 70-70, 2005.

[2] J.T. Cacioppo, L. Tassinary, and G. Berntson, "Handboook of psychophysiology," Cambridge university press, 2007.

[3] H. Kurniawan, A. V. Maslov, and M. Pechenizkiy, "Stress detection from speech and galvanic skin response signals," Proceedings of the 26th IEEE International Symposium on Computer-Based Medical Systems, pp. 209-214, 2013.

[4] L. Piwek, D. A. Ellis, S. Andrews, and A. Joinson, "The rise of consumer health wearables: promises and barriers," PLoS medicine, vol. 13, no. 2, 2016.

[5] J. W. Ahn, K. Yunseo, and C. K. Hee, "A novel wearable EEG and ECG recording system for stress assessment," Sensors, vol. 19, no. 9, 2019.

[6] S. Cohen, T. Kamarck, and R. Mermelstein, "A global measure of perceived stress," Journal of health and social behavior, pp. 385-396, 1983.

[7] T. D. Topolski, J. LoGerfo, D. L. Patrick, B. Williams, J. Walwick, and M. M. B. Patrick, "Peer reviewed: the Rapid Assessment of Physical Activity (RAPA) among older adults," Preventing chronic disease, vol. 3, no. 4, 2006.

[8] C. J. Golden, and S. M. Freshwater, "The Stroop Color and Word Test: A Manual for Clinical and Experimental Uses," Chicago, IL: Stoelting, 2002.

[9] D. Giokoumis, A. Drosou, P. Cipresso, D. Tzovaras, G. Hassapis, A. Gaggioli, and G. Riva, "Using activity-related behavioural features towards more effective automatic stress detection," PloS one, vol. 7, no. 9, 2012.

[10] J. A. Healey, and R. W. Picard, "Detecting stress during real-word driving tasks using physiological sensors," IEEE Transactions on intelligent transportation systems, vol. 6, no. 2, pp. 156-166, 2005.

[11] E. J. Peacok, and P. T. P. Wong, "The stress appraisal measure (SAM): a multidimensional approach to cognitive appraisal," Stress medicine, vol. 6, no. 3, pp. 227-236, 1990.

[12] R. Favilla, V. C. Zuccalà, G. Coppini, "Heart Rate and Heart Rate Variability from Single-Channel Video and ICA Integration of Multiple Signals," IEEE Journal of biomedical and health informatics, vol. 23, no. 6, pp. 23982408, 2018.

Address for correspondence:

Veronica Chiara Zuccalà.

Institute of Clinical Physiology of the National Research

Council (IFC - CNR).

Via Moruzzi 1, 56124 Pisa, Italy.

veronica.zuccala2@unibo.it. 